\title{
An enhanced genetic algorithm based courses timetabling method for maximal enrollments using maximum matching on bipartite graphs ${ }^{*}$
}

\author{
DUONG THANG LONG \\ Hanoi Open University, Vietnam \\ duongthanglong@hou.edu.vn
}

\begin{abstract}
Universities usually use academic credit systems for holding all training courses. They have to establish a suitable timetable for enrollment by students at beginning of every semester. This timetable must be met to all hard constraints and it is satisfied to soft constraints as high as possible. In some universities, students can enroll to the established timetable so that among of their courses is as much as possible. This leads to finish their studying program earlier than normally cases. In addition, this also leads to well-utilized resources such as facilities, teachers and so forth in universities. However, a timetable usually has so many courses and some its courses have same subjects but different time-slots. These may cause difficulties for manually enrolling by students. It may be fall into conflict of time when choosing two courses at same time-slots. It is difficult for enrollment with high satisfied. In this paper, we design a genetic algorithm based method for university timetable with maximal enrollments by using maximum matching on bipartite graphs.
\end{abstract}

Keywords: University timetables, genetic algorithm, bipartite graph, maximum matching.

\section{INTRODUCTION}

University timetabling is typical scheduling problem and it is also a classical problem. Universities usually use academic credit systems for holding all training courses. They have to establish a suitable timetable for enrolling by students at beginning of every semester. However, this problem has many complicated factors. They may be capable teaching and time inquired of teachers, a lot students, many kinds of classrooms and subjects, and especially major constraints within these elements. This problem also includes many relevant factors which should be considered such as examinations, practice, lecture halls, etc. Authors in [2-7] show that the timetabling problem is a kind of NP-hard. Typically, timetabling problems are conducted in traditional ways by intuitive and direct calculation of human. Currently, due to diversities and many relations between elements, this problem often takes a lot of time and labor. Using computers for dealing with this problem is not only much interesting to researchers, but also allowance achieving superior results despite many more constraints. Obviously, this leads to save a lot of time and effort.

Solving methods of this problem have been researched by many authors. In [6], authors

\footnotetext{
* This research is funded by Hanoi Open University.
} 
pointed out that, Hertz proposed using Tabu search with including two stages (TATI / TAG) and it is an appropriate method for scheduling problems with large-scale implementation. Nothegger suggests ant colony optimization (ACO) for solving this problem. Tassopoulos and Beligiannis use swarm optimization to establish a timetable for various schools in Greece. Al. Betar et al. propose a hybrid method (HHS) to solve scheduling problems for universities. HHS is an integrated algorithm with optimization and climbing hills swarm to balance space exploration and searching.

Due to efficiencies of genetic algorithms (GAs), a lot authors use GAs for timetable problems to improve performance of traditional methods. Authors in [2] indicated that GAs can be used as a properly universal method for complicated optimization problems, which they almost have no deterministic solution. Enhanced GA based methods can achieve high performance by adjusting genetic operations. Authors can use an alternative strategy in order to avoid falling into local optimum. In facts, M. Abbaszadeh in [2] used GA with changed structure of performing gene sequence which allows transferring $15 \%$ of better individuals to next generation in mutation operators. Moreover, to avoid falling into local optimum, they considered impact their parameters to mutations. They also removed repetitive genes and replaced by better gene sequences. Results of this method are high performance and maximum accuracy. Authors in [11] proposed a GA with binding elements of this problem to adapt practical constraints such as requirements of faculties for time, teaching expertise. They use fuzziness measurements in some genetic operations. In [1], we used hedge algebras based fuzziness measure for presenting school time of teachers. We also adjusted some genetic operations such as selection, crossover, mutation and replacement by using the temperature factor in simulated annealing. This has achievements of improving performance.

However, these authors mainly focus on how to improve efficiency of solutions with having no violation of hard constraints and maximal satisfied soft constraints. They do not consider resulted timetable which it gives the best case of enrollment for every student. There are two things can be treated well. Firstly, how to generate a good timetable so that students can have more opportunities of enrollment. Students can enroll as many courses as possible. Secondly, once a timetable is generated, how can students enroll suitable courses by their self so that they enroll courses as many as possible. In this paper, we propose enhanced GA based method for timetabling problems with maximal capability of enrollment. We use maximum matching on bipartite graphs to get maximal enrollment of every student. This article consists of 5 sections, Part 1 introductions to universities timetabling problems. Part 2 is detail of genetic algorithms based method for this problem. Part 3 proposes an enhanced GA based method with using maximum matching on bipartite graphs. Part 4 is about computer program and testing on real data in Hanoi Open University. The final section is conclusion.

\section{GENETIC ALGORITHS BASED METHOD FOR TIMETABLING PROBLEMS}

In general, course-based timetabling problems (CTP) consists of assigning appropriate timeslots, teachers and rooms to all given courses. This is done to ensure that all hard constraints 
are met and take satisfactions of soft constraints as high as possible. However, in academic credit training systems, it depends on characteristics of each university. CTP will be deployed with certain differences. Some universities let students enroll subjects firstly. Then they use these enrollments as parameters of the problem. They divide students into each class and fix them in every class. These classes are treated as resources and we assign classes to courses. So, all students of each class will be assigned to such courses. In this case, students cannot enroll for any further courses and they are always fixed in a class. This is not flexible for students and it does not give many choices for them such as time-slots, lecturers. In this paper, we use resources including time-slots, teachers and classrooms. This would be appropriate to the case that a timetable will be implemented before students enroll.

Steps for solving CTP can be described as follows: At the beginning of a semester, from learnable subjects of students, we propose all possible courses. Then we establish a timetable by assigning resources (time-slots, teachers and rooms) to every course. We announce generated timetable to students for enrolling. So, CTP problem consists of assigning teachers, time-slots, rooms to courses so that all hard constraints $(\mathrm{H})$ must be met and soft constraints (S) can be satisfied as much as possible.

In universities, hard and soft constraints are composed of various factors. They can be following requirements:

(H1) Each teacher or room is not assigned to more than one course at a time-slot.

(H2) Rooms must be assigned to appropriate courses. A practical room cannot be assigned to a theory course and vice versa, a hall room should not be assigned to small courses, etc.

(H3) Each teacher must be assigned to courses so that he or she has sufficient knowledge and capability of teaching for courses.

(H4) Teachers must be assigned to courses with time-slots so that they are present at the school. Each teacher has a list of time-slots for presenting at school.

(S1) Teachers are assigned to courses so that their expertise of courses is as high as possible.

(S2) Teachers are priority assigned to theirs expected time-slots as high as possible.

(S3) It should be to balance number of courses for every teacher, i.e., the minimum and maximum number of courses of every teacher should be taken.

(S4) It should be given priority courses with prerequisite of a subject to same time-slot. This aims to increase abilities of enrollments on the timetable.

(S5) Abilities of enrollment for every student on the timetable is as high as possible.

In this paper, we assume that it is not necessary to design curriculums with fixed mandatory subjects of every semester. Instead, we design a diagram of pre-requisite subjects for curriculums. When students want to choose subjects for learning in a semester, he or she must be passed all pre-requisite subjects belonging to the chosen subjects. For this case, soft constraints S4 and S5 have significant meanings. Soft constraint S4 means the more courses of same time-slot, the more chance of enrollment for students. However, these courses must be together pre-requisite. For S5 constraint, when students choose more subjects for learning then they can early graduated. In addition, if we reach high satisfied S5 then we many students at school in a semester. This means that school financing will be increasing, facilities are much used, etc.

Depending on particular academic credit training systems of a university, soft constraints 
can be adjusted some parameters for suitable reality. CTP can be formalized as an optimization problem model and we now describe its input data. In this model, we use following symbols:

- $\left\{C_{1}, C_{2}, \ldots, C_{n_{C}}\right\}$ denotes set of courses, $n_{C}$ is number of courses;

- $\left\{L_{1}, L_{2}, \ldots, L_{n_{L}}\right\}$ denotes set of teachers (lectures), $n_{L}$ is number of teachers;

- $\left\{R_{1}, R_{2}, \ldots, R_{n_{R}}\right\}$ denotes set of rooms, $n_{R}$ is number of rooms;

- $\left\{T_{1}, T_{2}, \ldots, T_{n_{T}}\right\}$ denotes set of time-slots, $n_{T}$ is number of time-slots;

- $\left\{S_{1}, S_{2}, \ldots, S_{n_{S}}\right\}$ denotes set of students, $n_{S}$ is number of students.

Normally, in universities, CTP should have weekly time-slots. A day of week can be divided into two sessions such as morning and afternoon. We assume that there are 6 days of a week from Monday to Saturday, then we have 12 time-slots of a week. However, we can also divide a day into many periods of time and weekly time-slots can be more than 12 .

In [1], we analysis these constraints in details, then H3, S1 and S3 constraints can be easily met by manually assigning teachers to every course based on experts. H1 constraint can be only obtained during progress of CTP by checking this constraint on a timetable. The remaining constraints are represented by matrices as following:

(H2) $C \times R=\left\{C R_{i, k} \mid i=\overline{1, n_{C}} ; k=\overline{1, n_{R}}\right\}$ defines constraints between rooms and courses. The value of this matrix is $\{0,1\}, C R_{i, k}=1$ implies that $R_{k}$ room can be assigned to $C_{i}$ course and 0 is not.

(H4, S2) $L \times T=\left\{L T_{j, l} \mid j=\overline{1, n_{L}} ; l=\overline{1, n_{T}}\right\}$ defines constraints between teachers and time-slots. We integrate $\mathrm{H} 4$ and S2 constraints. In which, H4 is strictly priority of time-slots for assigning with teachers and S2 is as high as possible. Therefore, this matrix will receive values in the form of language. For example, NO, NORMAL, GOOD, VERY GOOD, etc. NO value denotes a teacher being no present at schools during at that time-slot.

(S4) $C \times C=\left\{C C_{i_{1}, i_{2}} \mid i_{1}=\overline{1, n_{C}} ; i_{2}=\overline{1, n_{C}}\right\}$ describes prerequisite subjects between courses. It will receive binary values, 0 if there are not prerequisite of two subjects or 1 if there is a prerequisite subject of another. Courses with prerequisite subjects should be assigned at same time-slots in order to increase ability of enrollment.

(S5) $S \times C=\left\{S C_{u, i} \mid u=\overline{1, n_{S}} ; i=\overline{1, n_{C}}\right\}$ describes constraints between students and courses. It receives binary values, 1 if student $S_{u}$ can learn course $C_{i}$ and 0 is not.

Now we represent a timetable as following table. For this table, columns are courses and rows are lecturers, time-slots and rooms, corresponding.

\begin{tabular}{|l|c|c|l|l|l|l|}
\hline Courses & $C_{1}$ & $C_{2}$ & $\ldots$ & $C_{i}$ & & $C_{n_{C}}$ \\
\hline Lecturers & $L_{j 1}$ & $L_{j 2}$ & $\ldots$ & $L_{j i}$ & & $L_{j n_{C}}$ \\
Rooms & $R_{k 1}$ & $R_{k 2}$ & $\ldots$ & $R_{k i}$ & & $R_{k n_{C}}$ \\
Time-slots & $T_{l 1}$ & $T_{l 2}$ & $\ldots$ & $T_{l i}$ & & $T_{l n_{C}}$ \\
\hline
\end{tabular}

Table 1. Representation of the timetable

In this table, as above mentioned, we assign lecturers to every course in order to certainly 
satisfy requirements of $\mathrm{H} 3$ constraint. This is also to balance number of courses for every teacher. However, a course can only continue running if number of its enrolled students is enough large. This is a dynamic factor, so universities should take somehow to ensure that the number of deployed courses is as high as possible.

Underlying this, CTP could be condensed in a shorten form. We just need assigning timeslots and rooms to courses on a timetable. In [1], we use linguistic terms of hedge algebras for $L \times T$ matrix values. If $x$ denotes a term, then semantic quantitative function of $x-v(x)$ can be following triangle for satisfying measurement of $\mathrm{H} 4$ and S2 constraints. For example, Figure 1 describes satisfying measurement of three time-slots for a teacher.

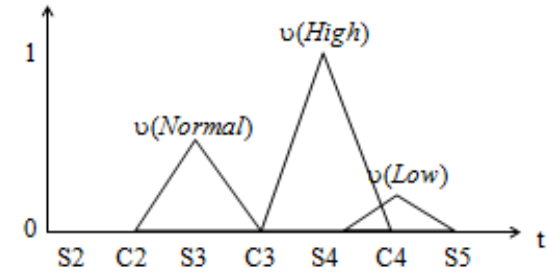

Figure 1. Selection of priority-time-slots of a teacher

In [1], for S2, we set $F_{k}^{2}$ being total time-satisfaction measure of $L_{k}$ teacher by evaluating satisfaction of assigned time-slots. S4 can be easily obtained by counting number of same timeslot assigned courses which they are together prerequisite. We set $F^{4}$ being totally this counting for measurement of S4 constraint. For S5, we evaluate it based on capability of enrollments according to $S \times C$ matrix. Once a timetable is generated, each student $S_{q}$ can enroll some courses for learning. Therefore, we can automatically build an enrollment solution for every student from $S \times C$ matrix and generated timetable. This solution can be obtained by applying optimal method which its objective is satisfaction of S5 as high as possible. However, this is a quite difficult sub-problem because of many factors and relations between elements. We will describe details in next section. From here, we just denote $F_{q}^{5}$ being satisfaction of enrollments. It is now can be stated a model of CTP as a formalization of multi-objectives optimization problems as follows:

$$
\left(\sum_{k=1}^{n_{L}} F_{k}^{2}\right) \rightarrow \max , \quad F^{4} \rightarrow \max , \quad\left(\sum_{q=1}^{n_{S}} F_{q}^{5}\right) \rightarrow \max
$$

subject to,

$$
\begin{aligned}
& \text { (H1.a) } \sum_{i=1}^{n_{C}} z_{i, l, j} \leq 1, \quad l=\overline{1, n_{T}}, j=\overline{1, n_{R}} \\
& \text { (H1.b) } \sum_{i=1}^{n_{C}} w_{i, k, l} \leq 1, \quad k=\overline{1, n_{L}}, l=\overline{1, n_{T}} \\
& \text { (H2) } \sum_{l=1}^{n_{T}} \sum_{j=1}^{n_{R}} C R_{i, j} \cdot z_{i, l, j}=1, \quad i=\overline{1, n_{C}} \\
& \text { (H4) } \sum_{k=1}^{n_{L}} \sum_{l=1}^{n_{T}} \omega\left(L T_{k, l}\right) \cdot w_{i, k, l}=1, \quad i=\overline{1, n_{C}}
\end{aligned}
$$

which $z_{i, l, j}$ is a binary variable for defining course $C_{i}$ be assigned to timeslot $T_{l}$ and classrooms $R_{j}$ if its value is 1 or otherwise. $\omega($.$) is a function to determine values of LT matrix,$ 
it may be NO $(\omega=0)$ or other linguistic terms $(\omega=1) . w_{i, k, l}$ is a binary variable for determining course $C_{i}$ be assigned to teacher $L_{k}$ and timeslot $T_{l}$ or not?

In [1], we proposed a genetic algorithm based method for solving CTP. This method uses temperature factors from simulated annealing (SA) as parameters for increasing convergence of the algorithm, and avoiding fall into local optimum as well.

a) Chromosome encoding

In general, for applying GA, we have to encode problem solutions into an appropriate gene sequence. By using directly encoding method, each gen of chromosome represents a parameter of solutions as a real number. For a timetable as in Table 1, as mentioned above, we manually assign teachers to every course by user expertise. Then, it need to encode parameters of rooms and time-slots, thus, a chromosome has $2 n_{C}$ length of gens as in Figure 2.

\begin{tabular}{|c|c|c|c|c|c|c|c|c|c|}
\hline \multicolumn{5}{|c|}{ Segment T: timeslots } & \multicolumn{5}{|c|}{ Segment R: classrooms } \\
\hline 0 & 1 & 2 & $\ldots$ & $n_{C}$ & 0 & 1 & 2 & $\ldots$ & $n_{C}$ \\
\hline & & & & & & & & & \\
\hline
\end{tabular}

Figure 2. Chromosome of gene encoding of solution

Value of each gene $\left(g_{i}\right)$ is real number in $[0,1]$, thereby we determine value of real domain of time-slots and rooms as well, by following functions $\left(f^{T}\right.$ and $\left.f^{R}\right)$ :

$$
\begin{aligned}
& f^{T}:[0,1] \rightarrow\left[1, n_{T}\right], f^{T}\left(g_{i}\right)=\left\lceil g_{i} \cdot n_{T}\right\rceil \\
& f^{R}:[0,1] \rightarrow\left[1, n_{R}\right], f^{T}\left(g_{i}\right)=\left\lceil g_{i} . n_{R}\right\rceil
\end{aligned}
$$

in which, symbols $\lceil$. 7 being the nearest upper integer of values.

b) Fitness function designing

We design a fitness function by integrating hard constraints and soft constraints. In directly encoding, we use penalty coefficients in fitness function to avoid violations hard constraints and get high satisfaction of soft constraints. Objectives of problems can be converted to minimization. In this case, we divide fitness function into two parts of soft and hard constraints. First part is satisfying measurement of soft constraints, it can be formulated as following function:

$$
F^{S}=w_{2} \cdot\left(\frac{1}{n_{L}} \sum_{k=1}^{n_{L}}\left(1-F_{k}^{2}\right)\right)+w_{3} \cdot\left(F^{4}\right)^{-1}+w_{4} \cdot\left(F^{5}\right)^{-1}
$$

where, $w_{1}, w_{2}$ and $w_{3}$ are weights of soft constraints in objective function.

Second part is measurement of violation hard constraints, it is can be formulated as following function:

$$
F^{H}=w_{1} \cdot\left(1-\frac{1}{1+c\left(H_{1}\right)+c\left(H_{2}\right)+c\left(H_{4}\right)}\right)
$$

where, $c($.$) is a function of counting violations number of hard constraints \mathrm{H} 1, \mathrm{H} 2, \mathrm{H} 4$.

The fitness function is weighted sum of $F^{H}$ and $F^{S}$ as following:

$$
\text { fitness }=F^{H}+F^{S}
$$

In this case, hard constraints can be met and fulfilled by setting weight of $F^{H}$ is much greater than others $\left(F^{S}\right)$. It means that soft constraints may be satisfied after eliminating 
violations of hard constraints.

c) Genetic operators implementation

We use genetic operations with integrated temperature $T_{k}$ as an additional parameter (where $k$ is the index of current generation). The probability for selection and mutation operations is changed through each generation by applying this temperature [1]. Genetic operations of selection, crossover, mutation and replacement are designed in [1,8] for generating new chromosomes during evolution.

In facts, the better timetable, the more enrollments of students. In order to get a good timetable, beside partly $F^{2}$ and $F^{4}$ of fitness function, we should consider details of $F^{5}$ because it causes the satisfaction of enrollments on a timetable. Thus, in next section, we apply maximum matching on bipartite graph for solving sub-problem of maximum enrollment.

\section{MAXIMIZE ENROLLMENTS USING MAXIMUM MATCHING ON BIPARTITE GRAPH}

In objectives of CTP, we have to maximize enrollments on a timetable. In [1], our method can reach high results of these objectives during evolution. However, there is an optimal subproblem in the last objective of CTP. It was not solved in our method because of quite difficult. In facts, once a timetable is generated, every student enrolls courses based on subjects that he or she can learn. This sub-problem become determining maximal courses that each student can enroll.

In general, at the beginning of a semester, we get all subjects which can be enrolled for every student. For each subject, we get number of students which can enroll for learning. Then, we propose all possible courses of every subject and put them into a table as following:

\begin{tabular}{|l|c|c|l|c|l|c|}
\hline Courses & $C_{1}$ & $C_{2}$ & $\ldots$ & $C_{i}$ & & $C_{n_{C}}$ \\
\hline Subjects & $S u b_{1}$ & $S u b_{2}$ & $\ldots$ & $S u b_{i}$ & & $S u b_{n_{C}}$ \\
\hline
\end{tabular}

Table 2. Proposed courses with corresponding subjects

where, a subject can belong to more than one course, i.e. $C_{i} \neq C_{j} \wedge S u b_{i}=S u b_{j}$. Students want to learn a subject, they can enroll a course belonging to this subject. Constraints between students and courses are represented in $S \times C$ matrix. Since $E_{q}^{5}$ denotes number of enrolled courses by a student $S_{q}$, we have to generate a timetable so that every student can get maximum $E_{q}^{5}$

For each student $S_{q}$, we build a bipartite graph which left side of vertices are subjects and right ones are time-slots (Figure 3). Firstly, we determine subjects that student $S_{q}$ can learn based on generated timetable. Then, each of these subjects is connected to time-slots which they are assigned to courses of this subject. These connections are edges of the bipartite graph as following picture: 


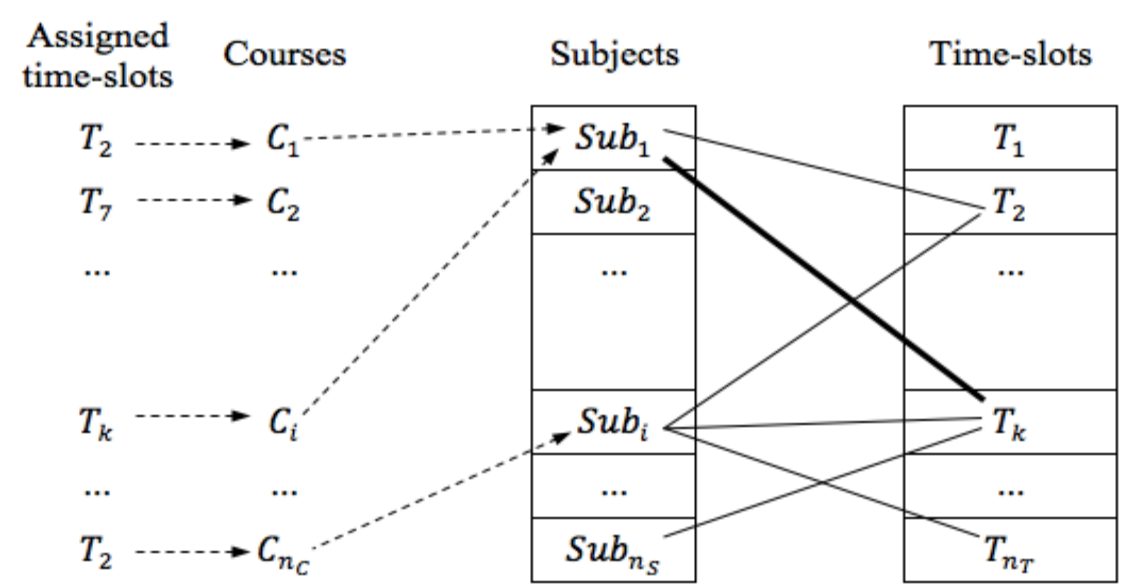

Figure 3. Bipartite graph for enrolling of a student

A time-slot may be assigned to more than one course in a timetable, so we have connections between a time-slot and many subjects. Furthermore, a subject may belong to more than one course and a time-slot is assigned to one course, we also have connections between a subject and many time-slots. For example, in Figure 3, time-slot $T_{k}$ is connected to three subjects of $S u b_{1}, S u b_{i}$ and $S u b_{n_{S}}$ or $S u b_{1}$ is connected to two time-slots of $T_{2}$ and $T_{k}$ because of subject $S u b_{1}$ belongs to two courses of $C_{1}$ and $C_{i}$.

Base on this bipartite graph, enrollments of a student can be reached by finding a set of edges which each edge indicates an enrollment of a subject and the corresponding time-slot. However, these edges have no common vertices, i.e. a student can only enroll a subject and a time-slot at once in a semester. For example, in Figure 3, if a student enrolls subject $S u b_{1}$ with corresponding $T_{k}$ (the bold line) then he or she cannot enroll $S u b_{1}$ and $T_{k}$ any more. This suggests that we can apply maximum matching methods on bipartite graphs to solve this subproblem, the more edges we find, the more subjects that student $S_{q}$ can enroll for learning.

We now can let $F_{q}^{5}$ be number of possible enrolled subjects by student $S_{q}$. With maximum matching of every student, we can get total satisfactions of enrollments for all students as following:

$$
F^{5}=\sum_{q=1}^{n_{S}} F_{q}^{5}
$$

In maximum bipartite matching, a set of edges with no common vertices is called a matching - $M$, thus this problem become finding $M$ with maximum cardinality. We apply Hopcroft-Karp algorithm for this problem [\#1]. A vertex has two statuses of matched and unmatched, and an edge is also matched or unmatched. It is said that an alternating path of $M$ is a path of a graph so that it has interleaved matched edge and unmatched edge in turn of $M$. We also call an augmenting path with respect to $M$ if it is an alternating path of $M$ with two endmost unmatched edges. For example, in Figure 4, the red edges are matched, we have three alternating paths but there is only one augmenting path at the middle (4.b) of this Figure.

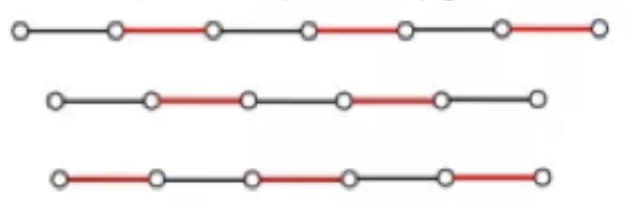

Figure 4. Alternating paths and augmenting paths 
It is known that If $M$ is a matching and $P$ is an augmenting path with respect to $M$, then $M \oplus P$ is a matching containing one more edge than $M$. In which, we use notation $A \oplus B$ to denote symmetric difference of two sets $\mathrm{A}$ and B, i.e. a set of all elements so that each element belongs to only one of two sets. For example, the augmenting path (4.b) with respect to $M$ (two red edges), we can get new one $M \oplus P$ with one more edge than $M$ (on the right of Figure 5).

Figure 5. The new augmenting path with one more edge

In addition, a matching $M$ in a graph $G$ is a maximum cardinality matching if and only if it has no augmenting path.

The Hopcroft-Karp algorithm is based on that each time for searching augmenting paths, instead of finding an augmenting path, we find a blocking set of augmenting paths with respect to $M$ which called $\left\{P_{1}, P_{2}, \ldots, P_{k}\right\}$ so that they are vertex-disjoint. Then we extend $M$ by applying $M \oplus P_{1} \oplus P_{2} \ldots \oplus P_{k}$. This procedure is repeated until no augmenting path exists.

Next section, we develop a computer program for our proposed method, then it is tested on an examples and real data sets in Faculty of Information Technology - Hanoi Open University.

\section{COMPUTATIONAL EXPERIMENTS}

\subsection{Experiment with sample problems}

We assume that a generated timetable in following table with 6 courses, 3 subjects, 4 teachers, 3 time-slots, 3 rooms and 3 students.

\begin{tabular}{l|c|c|c|c|c|c|}
\multicolumn{1}{l}{ Subjects } & $S u b_{1}$ & $S u b_{2}$ & $S u b_{1}$ & $S u b_{1}$ & $S u b_{3}$ & $S u b_{2}$ \\
\hline Courses & $C_{1}$ & $C_{2}$ & $C_{3}$ & $C_{4}$ & $C_{5}$ & $C_{6}$ \\
\hline Teachers & $L_{1}$ & $L_{2}$ & $L_{1}$ & $L_{3}$ & $L_{4}$ & $L_{2}$ \\
\hline Time-slots & $T_{1}$ & $T_{1}$ & $T_{2}$ & $T_{3}$ & $T_{1}$ & $T_{2}$ \\
\hline Rooms & $R_{1}$ & $R_{2}$ & $R_{1}$ & $R_{1}$ & $R_{3}$ & $R_{2}$ \\
\hline
\end{tabular}

Table 3. The sample timetable with corresponding subjects

For illustrating maximum number of possible enrollments, we leave out all constraints matrix unless $S \times C$ matrix (Table 4). Three students $S_{1}, S_{2}$ and $S_{3}$ can learn $\left\{S u b_{2}, S u b_{3}\right\}$, $\left\{S u b_{1}, S u b_{2}, S u b_{3}\right\}$ and $\left\{S u b_{1}, S u b_{3}\right\}$, respectively.

\begin{tabular}{|c|c|c|c|c|c|c|}
\multicolumn{1}{c}{ Subjects } & $S u b_{1}$ & $S u b_{2}$ & $S u b_{1}$ & $S u b_{1}$ & \multicolumn{1}{c}{$S u b_{3}$} & $S u b_{2}$ \\
\hline $\begin{array}{c}\text { Courses } \\
\text { Students }\end{array}$ & $C_{1}$ & $C_{2}$ & $C_{3}$ & $C_{4}$ & $C_{5}$ & $C_{6}$ \\
\hline$S_{1}$ & 0 & 1 & 0 & 0 & 1 & 1 \\
\hline$S_{2}$ & 1 & 1 & 1 & 1 & 1 & 1 \\
\hline$S_{3}$ & 1 & 0 & 1 & 1 & 1 & 0 \\
\hline
\end{tabular}

Table 4. Constraints between students and courses $(S \times C)$ 
We build three bipartite graphs as in Fig. 6, Fig. 7 and Fig. 8 for students $S_{1}, S_{2}$ and $S_{3}$, respectively.

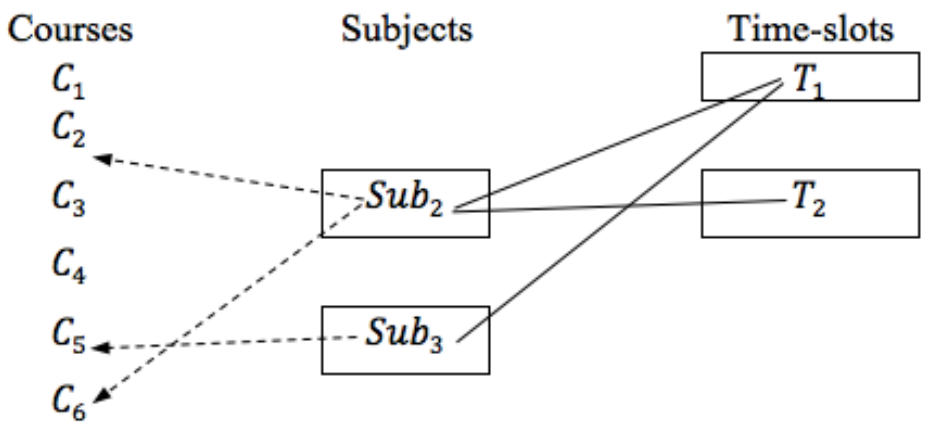

Figure 6. Bipartite graph for student $S_{1}$

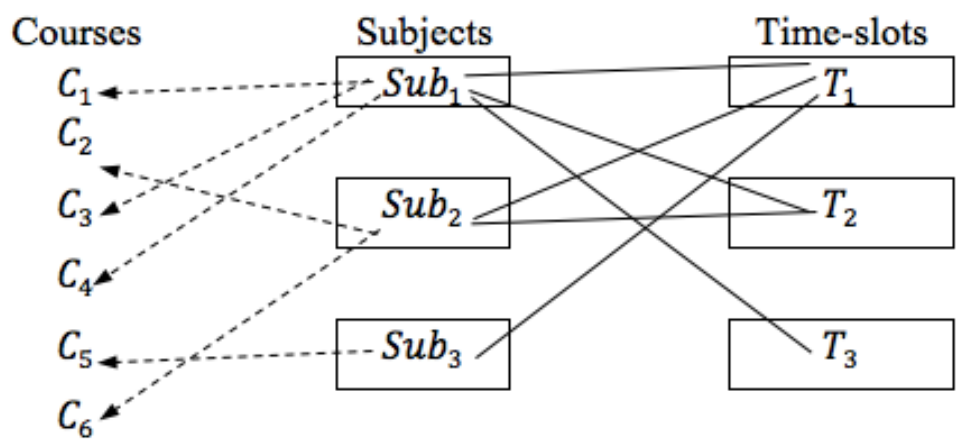

Figure 7. Bipartite graph for student $S_{2}$

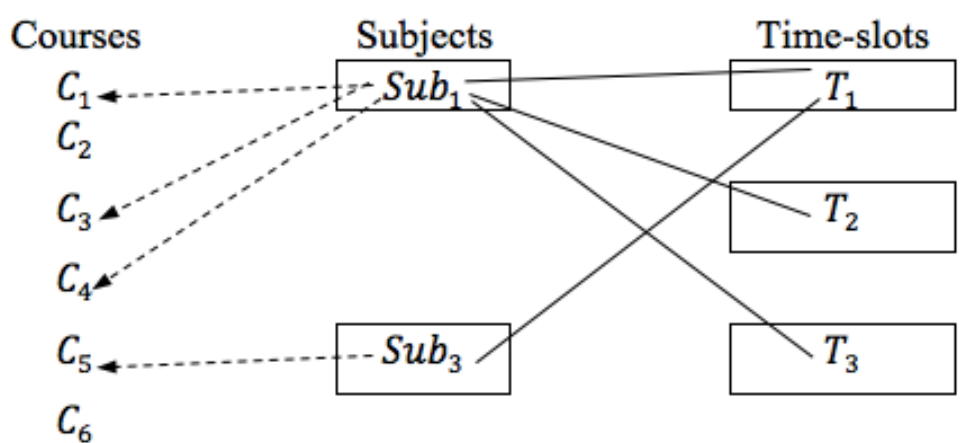

Figure 8. Bipartite graph for student $S_{3}$

For maximum matching of these graphs, in this simple case, we manually determine $M_{1}=$ $\left\{\left(S u b_{2}, T_{2}\right),\left(S u b_{3}, T_{1}\right)\right\} \quad, \quad M_{2}=\left\{\left(S u b_{1}, T_{3}\right),\left(S u b_{2}, T_{2}\right),\left(S u b_{3}, T_{1}\right)\right\} \quad$ and $\quad M_{3}=$ $\left\{\left(S u b_{1}, T_{2}\right),\left(S u b_{3}, T_{1}\right)\right\}$ for students $S_{1}, S_{2}$ and $S_{3}$ respectively.

In facts, in Fig. 6, if we choose an edge of $\left(S u b_{2}, T_{1}\right)$ then there is no more edge can be chosen, so it is not a maximum matching for student $S_{1}$. Similarly, in Fig. 7 and Fig. 8, if we choose the edge of $\left(S u b_{1}, T_{1}\right)$, then there is no more edge can be chosen for $S u b_{3}$ subject, in this case, we cannot reach to a maximum matching for student $S_{2}$ and $S_{3}$. In Table 5, we give all matching of each student, it shows that which one is maximum matching. The largest number of possible learning subjects for a student is number of edges in maximum matching. The maximum matching also indicates subjects and time-slots for enrollments of 
corresponding student.

\begin{tabular}{|c|c|c|c|}
\hline $\begin{array}{c}\text { Fig. of student } \\
\text { Number of edges }\end{array}$ & Fig. 6 of $S_{1}$ & Fig. 7 of $S_{2}$ & Fig. 8 of $S_{3}$ \\
\hline 1 & $\left(S u b_{2}, T_{1}\right)$ & $\left(S u b_{1}, T_{2}\right)$ & $\left(S u b_{1}, T_{1}\right)$ \\
\hline 2 & $\begin{array}{c}\left(S u b_{2}, T_{2}\right) \\
\left(S u b_{3}, T_{1}\right)\end{array}$ & $\begin{array}{l}\left(S u b_{1}, T_{1}\right) \\
\left(S u b_{2}, T_{2}\right)\end{array}$ & $\begin{array}{l}\left(S u b_{1}, T_{2}\right) \\
\left(S u b_{3}, T_{1}\right)\end{array}$ \\
\hline & & $\begin{array}{l}\left(S u b_{1}, T_{3}\right) \\
\left(S u b_{2}, T_{2}\right) \\
\left(S u b_{3}, T_{1}\right)\end{array}$ & \\
\hline
\end{tabular}

Table 5. Matching with its size for each bipartite graph of students

\subsection{Experiment with a real-world problem}

In [1], we use a real-world dataset of Faculty of Information Technology - Hanoi Open University. The detail of this dataset is also showed in [1]. In this paper, we summarize dataset and parameters for running in Table 6. We establish an experiment running with plugged maximum bipartite matching into fitness function of GA for getting the largest number of possible learning subjects.

Name of parameters
\begin{tabular}{|l|c|}
\hline Number of lecturers & 75 \\
\hline Number of time-slots & 20 \\
\hline Number of rooms & 15 \\
\hline Number of students & 1044 \\
\hline Number of subjects & 42 \\
\hline Number of courses & 86 \\
\hline $\boldsymbol{\alpha}-$ Temperature decreasing factor in genetic & 0.7 \\
\hline operations & 9 \\
\hline $\boldsymbol{\gamma}_{m a x}-$ Maximal temperature in genetic operations & 250 \\
\hline$N_{p o p}-$ Population size & 1000 \\
\hline$G_{m a x}-$ Maximal generation in evolution & 0.9 \\
\hline$p_{c}-$ Crossover probability & 0.1 \\
\hline$p_{m}-$ Mutation probability & $(0.9,0.01$, \\
\hline$\left(W_{1}, W_{2}, W_{3}, W_{4}\right)-$ Weights of components in fitness & $0.01,0.08)$ \\
\hline
\end{tabular}

Table 6. Summary dataset of running experiments 
By directly encoding, an individual is represented for a timetable. We use maximum bipartite matching for counting number of learnable subjects based on individuals. So, values of $F^{5}$ is evaluated for fitness function. Then, we compute the total number of subjects for all students and get maximum, minimum and average of them in all individuals of each generation. These are compared with the version of no applying maximum bipartite matching in [1].

We run this experiment in three times which are denoted by Run1, Run2, Run3. The number violations of hard constraints are decreasing from about nearly 100 down to zero at about $850^{\text {th }}$ generation for all three running times (Figure 9). We set high weighting for avoiding violations of hard constraints, it is set by 0.9. Number violations of hard constraints are much decreasing at early generations of evolution, then it is kept decreasing in priority to zero.

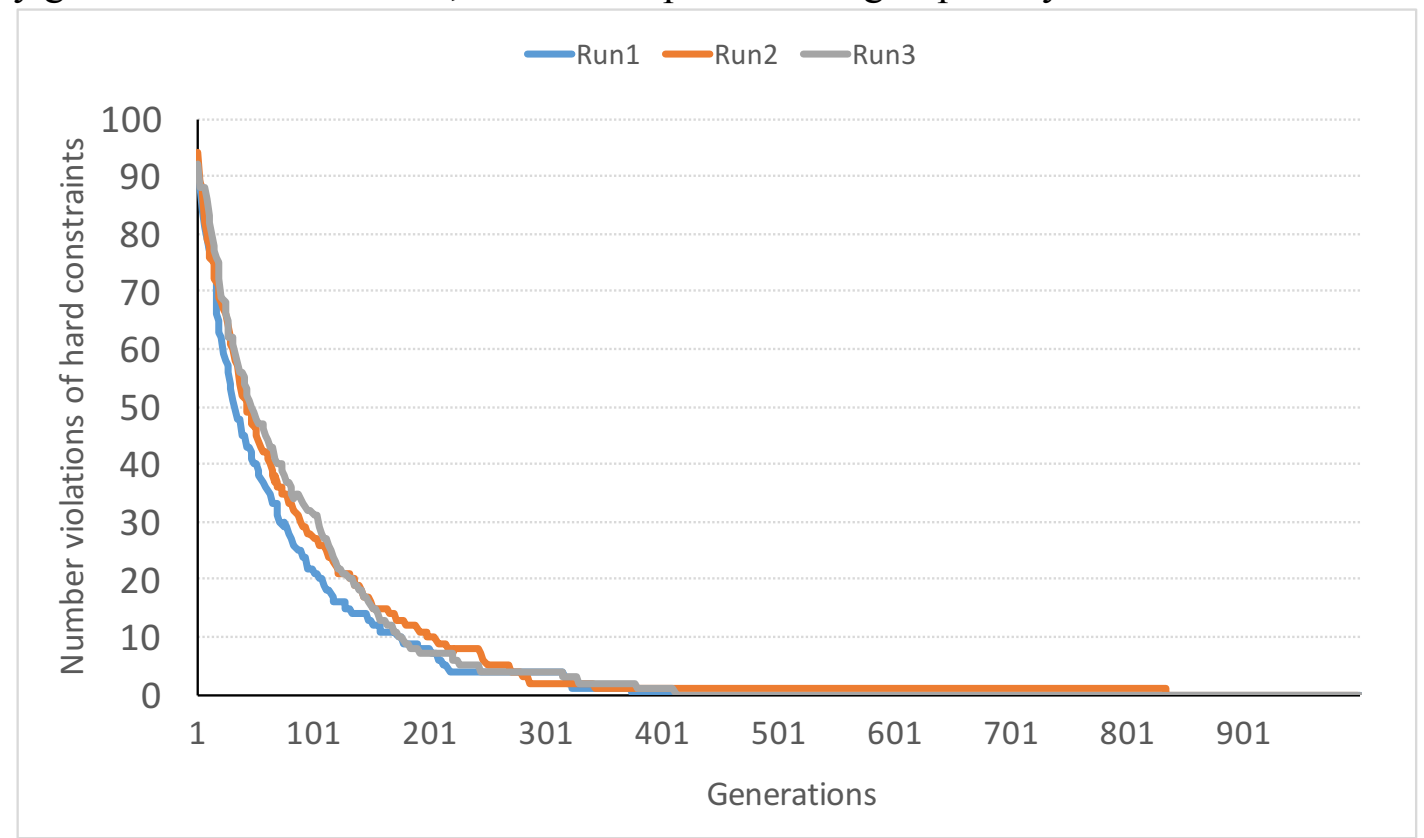

Figure 9. Number violations of hard constraints decreasing in generations

Number of all possible enrollments of the best individual in every generation is showed in Figure 10. For early generations, this number is quite high due to there are some violations of hard constraints. It is also quite much changed at each generation after that, and stability of this number is reached at about $800^{\text {th }}$ generation. 


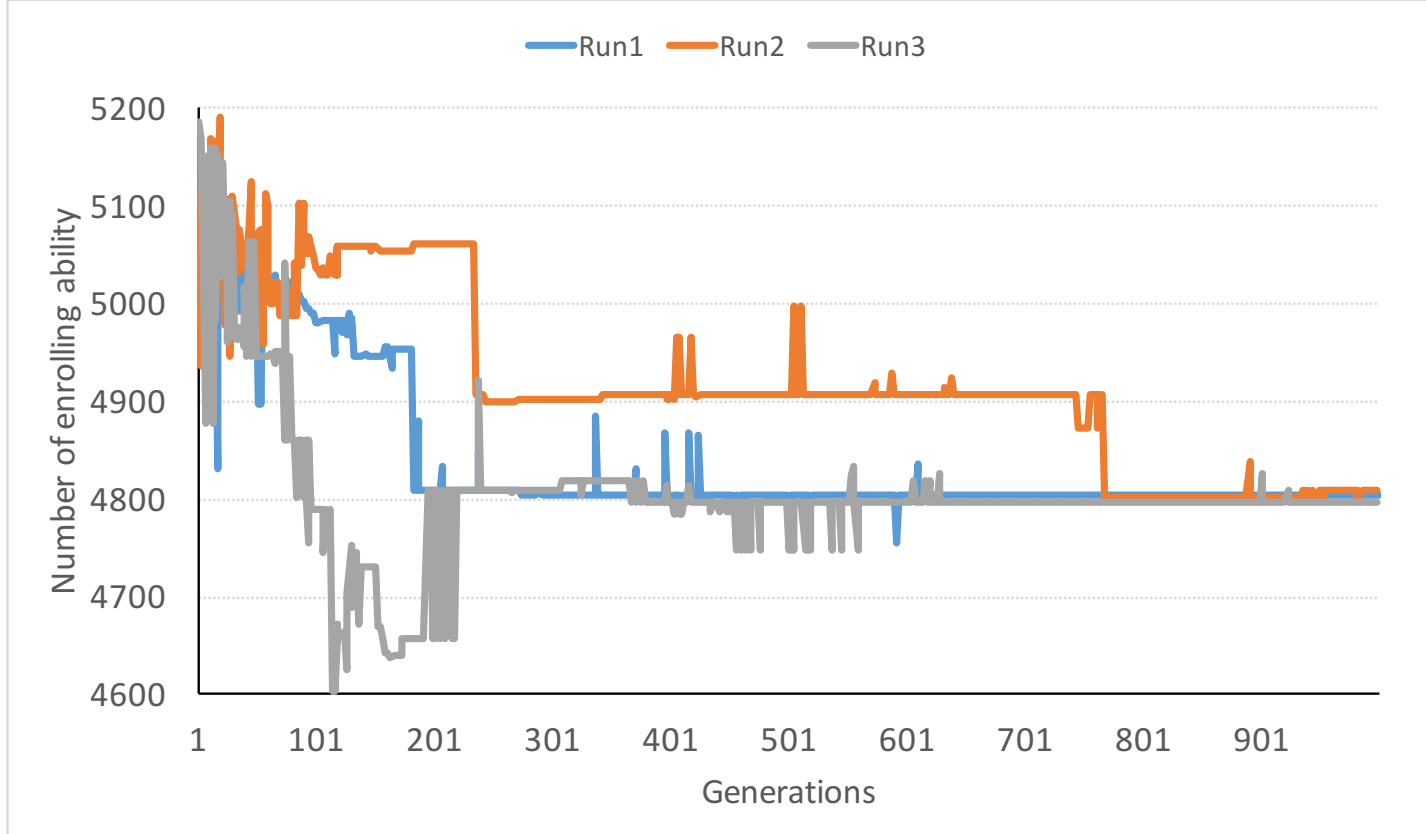

Figure 10. Number of possible enrollments of the best individual in generations

The most important result in this paper is number of possible enrollments of the best individual in each experiment running. In all running, this number is higher than our method in [1] which it does not apply maximal bipartite matching for evaluating possible enrollments of students on timetables. In Figure 11, this number of first, second and last running is higher than [1] by $51,144,143$, respectively.

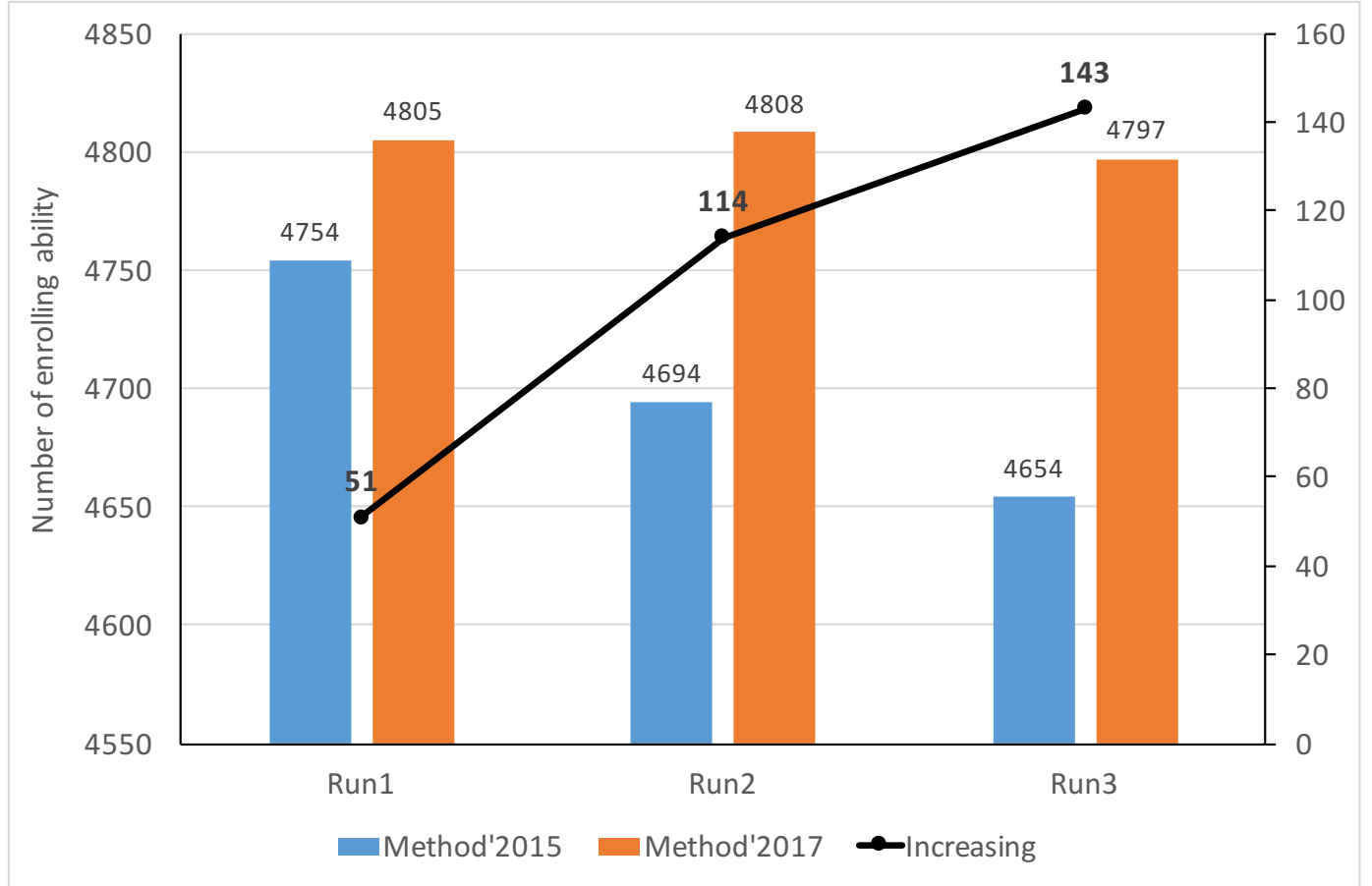

Figure 11. Number of enrolling ability of the best individual in generations

For this result, it shows that the good performance of our enhanced method in number of possible enrollments for students. This can make decreasing number of canceled courses when 
a generated timetable is used for enrolling by students, because, it gives more chances for enrolling and we use maximal enrollments of every student for suggestion in reality.

\section{CONCLUSION}

In this paper, we propose a genetic algorithm based method for timetable problems in credits training at universities. Especially, we utilize maximal matching on bipartite graph for a suboptimization problem in genetic algorithms, which is maximal enrollments of students.

The results of experiments in practice at the Faculty of Information Technology - Hanoi Open University show effectiveness of our proposed method. Running time of this algorithm is much faster than before, in comparing to traditional methods, it reduces much time to obtain the final timetable. It takes about 15 minutes while traditional method takes about 2 weeks by manually done. Moreover, results of this method show that final timetable gives much more opportunities for enrollments by students. Students also easily enroll courses for learning based on suggestions of maximal enrollments which is outputted by this algorithm. These also show potential effectiveness of this algorithm in practical application.

The proposed method in this paper can be extended to apply for practicing in many situations of credit courses training in universities. However, hard constraints and soft constraints may be considered more different assessments to show suitable of each assessment. Especially, we can use fuzzy parameters with more suitable for purpose of actual use, thereby efficiency is potentially increased. These will be studied further and announced in next research. 


\section{REFERENCES}

[1] Duong Thang Long, "A genetic algorithm based method for university course timetabling problems and application in Hanoi Open University", Journal of Computer Science and Cybernetics, Vol.32, No.4, pp.285-301, 2016.

[2] M.Abbaszadeh and S.Saeedvand, "A Fast Genetic Algorithm for Solving University Scheduling Problem", IAES International Journal of Artificial Intelligence (IJ-AI), Vol. 3, No. 1, pp. 7-15, 2014.

[3] A.O.Modupe et al, "Development of a University Lecture Timetable using Modified GA Approach", International Journal of Advanced Research in Computer Science and Software Engineering, Vol. 4, Iss. 9, pp. 163-168, 2014.

[4] W.Chinnasri et all, "The Suitable Genetic Operators for Solving the University Course Timetabling Problem", Journal of Convergence Information Technology (JCIT), Vol. 8, No. 12, pp. 60-66, 2013.

[5] S.R.Sutar \& R.S.Bickar, "University Timetabling based on Hard Constraints using GA", International Journal of Computer Applications (0975 - 8887), Vol.42, No.15, pp. 1-5, 2012.

[6] M.S.Kohshori et al, "Hybrid Genetic Algorithm for University Course Timetabling", IJCSI International Journal of Computer Science Issues, Vol. 9, Issue 2, No 2, pp. 446-455, 2012.

[7] K.Kumar et al, "Genetic Algorithm Approach to Automate University Timetable", International Journal of Technical Research(IJTR), Vol 1, Issue 1, pp.47-51, 2012.

[8] Cat Ho Nguyen, Witold Pedrycz, Thang Long Duong, Thai Son Tran, "A genetic design of linguistic terms for fuzzy rule based classifiers", International Journal of Approximate Reasoning, Vol.54, pp.1-21, 2012.

[9] Rahul Malhotra, Narinder Singh \& Yaduvir Singh, "Genetic Algorithms: Concepts, Design for Optimization of Process Controllers”, Computer and Information Science, Vol. 4, No. 2, pp. 3954, 2011.

[10] Vasileios Kolonias et al, "Solving the Examination Timetabling Problem in GPUs", Algorithms, Vol.7, pp.295-327, 2014.

[11] Radomir Perzina and Jaroslav Ramik, "Timetabling Problem with Fuzzy Constraints: A SelfLearning Genetic Algorithm", International Journal of Engineering and Innovative Technology, Vol.3, Iss.4, 2013. 